\title{
Effect of pulsed transcranial ultrasound stimulation at different number of tone-burst on cortico-muscular coupling
}

\author{
Ping Xie* ${ }^{*}$, Sa Zhou, Xingran Wang, Yibo Wang and Yi Yuan*
}

\begin{abstract}
Background: Pulsed transcranial ultrasound stimulation (pTUS) can modulate the neuronal activity of motor cortex and elicit muscle contractions. Cortico-muscular coupling (CMC) can serve as a tool to identify interaction between the oscillatory activity of the motor cortex and effector muscle. This research aims to explore the neuromodulatory effect of low-intensity, pTUS with different number of tone burst to neural circuit of motor-control system by analyzing the coupling relationship between motor cortex and tail muscle in mouse. The motor cortex of mice was stimulated by pulsed transcranial ultrasound with different number of tone bursts (NTB=100 150200250 300). The local field potentials (LFPs) in tail motor cortex and electromyography (EMG) in tail muscles were recorded simultaneously during pTUS. The change of integral coupling strength between cortex and muscle was evaluated by mutual information (MI). The directional information interaction between them were analyzed by transfer entropy (TE).

Results: Almost all of the MI and TE values were significantly increased by pTUS. The results of MI showed that the CMC was significantly enhanced with the increase of NTB. The TE results showed the coupling strength of CMC in descending direction (from LFPs to EMG) was significantly higher than that in ascending direction (from EMG to LFPs) after stimulation. Furthermore, compared to NTB $=100$, the CMC in ascending direction were significantly enhanced when $N T B=250,300$, and $C M C$ in descending direction were significantly enhanced when $N T B=200,250,300$.

Conclusion: These results confirm that the $\mathrm{CMC}$ between motor cortex and the tail muscles in mouse could be altered by pTUS. And by increasing the NTB (i.e. sonication duration), the coupling strength within the cortico-muscular circuit could be increased, which might further influence the motor function of mice. It demonstrates that, using MI and TE method, the CMC could be used for quantitatively evaluating the effect of pTUS with different NTBs, which might provide a new insight into the effect of pTUS neuromodulation in motor cortex.
\end{abstract}

Keywords: Pulsed transcranial ultrasound stimulation, Cortico-muscular coupling, Number of tone bursts, Mutual information, Transfer entropy

\section{Background}

Neuromodulation techniques have gained attention recent years for both neuroscientific research and neural engineering applications $[1,2]$. Pulsed transcranial ultrasound stimulation (pTUS) $[3,4]$ is a promising technique for neuromodulation which has non-invasiveness, high spatial resolution $(<2 \mathrm{~mm})$, and deep penetration [5-7].

*Correspondence: pingx@ysu.edu.cn; yuanyi513@ysu.edu.cn Institute of Electric Engineering, Yanshan University,

Qinhuangdao 066004, Hebei, China
As a mechanical pressure wave, pulsed ultrasound can be transmitted through the skull and facilitate or inhibit neural activities $[8,9]$. By observing the cerebral blood flow [10], LFPs or EEG signals from brain [11,12] or electromyography (EMG) signals from the muscle [13-15], etc., the effect of pTUS have been widely investigated. For instance, Legon $\mathrm{W}$ et al. modulated the activity of primary somatosensory cortex and spectral content of sensory-evoked brain oscillations in humans [16]. Li [10] and Guo [17] used low-intensity pTUS to modulate the brain of stroke rats and found pTUS is neuroprotective 
for ischemic brain injury. Previously, we [11] found that the focused ultrasound stimulation could modulate the phase-amplitude coupling between neuronal oscillations in the rat hippocampus. Moreover, pTUS can stimulate the motor cortex to induce muscle contraction and EMG signals [13]. These rapidly increasing body of findings provide ample evidence that ultrasound stimulation can flexibly modulate the cortical oscillatory dynamics and induce evident motor response.

As a well-established neurophysiological measure, cortico-muscular coupling (CMC) can be used to understand the communication between the oscillation of the cortical and spinal cord activities [18-20]. It is generally believed that the effective movement control depends on the synchronization of oscillatory activity between the motor cortex and effector muscle [21, 22]. By analyzing the coupling between the local field potentials (LFPs) (or magnetoencephalogram (MEG), electroencephalogram (EEG)) of the motor cortex and the electromyogram (EMG) of muscles, previous studies shown that CMC is related to the motor performance [23] and could identify the impaired neural pathway in patients [24]. As pTUS could elicit evident muscle contraction [13] and modulate neural oscillatory [11], we speculate that pTUS-induced change of information flow between motor cortex and effector muscle is subsistent, which could be evaluated by CMC. Previous studies about the effect of pTUS mainly focus on the change of neural activities in the brain [25] or the motor response in muscle [26], however, the coupling between the cortical and spinal cord activities during pTUS is still unknown. Therefore, it is important to evaluate the influence of pTUS with different parameters on neuromodulation from a cortical-muscular coupling view.

As the neural network of cortico-muscular system has nonlinear features of its parts and interactions between them [27], MI [28] and TE [29], which are model-free and sensitive to nonlinear interaction [30], are capable of quantitatively describing the cortico-muscular coupling by measuring the statistical dependencies between two variables [31-33]. In addition, the coupling between cortical and the targeted muscle is bidirectional which includes both the motor command from the cortex and feedback information from the contracting muscle [34, 35]. Because MI is symmetric, it could be used to quantify the amount coupled information of cortico-muscular [33] without the directional information between them [36]. TE which complements the non-directional defect of MI [37], can be used to evaluate the directional interaction of CMC [32].

In the present study, we introduce a novel way to assess the effect of pTUS with different NTBs by applying the cortico-muscular coupling between motor cortex and the tail muscles in mice, thus allowing for quantification of ultrasound effect on motor command circuit. First, since low-intensity pTUS is capable of neuromodulation without thermal effects or tissue damage $[8,38]$, the lowintensity transcranial ultrasound $\left(1.1 \mathrm{~W} / \mathrm{cm}^{2}\right)$ was applied to stimulate the motor cortex in mice at different number of tone bursts $(\mathrm{NTB}=100,150,200,250,300)$. Then, the LFPs in tail motor cortex and EMG in tail muscles were recorded simultaneously during pTUS. Finally, based on the recorded LFPs and EMG signals, the integral coupling strength between cortex and muscle induced by pTUS was evaluated by mutual information (MI), and the change of directional information interaction between them was analyzed using and transfer entropy (TE).

\section{Methods \\ Data recording \\ Experimental system and parameter settings}

The experimental system is shown in Fig. 1a, consisting of six main components: (1) two function generators (AFG3022C, Tektronix, USA), (2) a linear radio frequency power amplifier (RFA) (240L, ENI Inc., USA), (3) an unfocused ultrasound transducer (V301-SU, Olympus, Japan) with center frequency of $500 \mathrm{kHz}$ and diameter of $31 \mathrm{~mm}$ driven by RFA, and (4) an custom conical plastic collimator (Length $50 \mathrm{~mm}$, diameter 2 and $31.2 \mathrm{~mm}$ ) filled with degassed ultrasound gel and delivering the pTUS to the cortex, (5) single-channel microelectrodes (WE50030.1B10, MicroProbe, USA) recording the LFPs and fine wire electrode recording EMG signals,(6) a dual-channel front-end amplifier (63386, A-M SYSTEMS INC., USA) that amplifying the LFPs and EMG signals, and a 16-channel neural signal processor (NSP) (Cerebus Data Acquisition System, Blackrock Microsystems, USA) converting the signals into digital signals, (7) a computer for data storage and displaying the recorded data simultaneously.

Ultrasonic parameters are illustrated in Fig. 1c, i.e., acoustic intensity (AI), the number of acoustic cycles per pulse $(\mathrm{NC})$, pulse repetition frequency (PRF), the number of tone bursts (NTB), the inter trial interval (ITI) and the sonication duration. In this paper, the parameter setting is $\mathrm{AI}=1.10 \mathrm{~W} / \mathrm{cm}^{2}, \mathrm{NC}=250, \mathrm{PRF}=1 \mathrm{kHz}, \mathrm{ITI}=3.6 \mathrm{~s}$. The excitability or inhibition of pTUS on the neural oscillatory activity are related to the ultrasound beam and parameters of ultrasound [17], especially the pulse repetition frequency. Based on our experiments and other literatures $[10,17,39]$, we used $P R F=1 \mathrm{kHz}$ to facilitate the motor cortical activity and evoke EMG signals in tail muscle. To explore the effect of pTUS to cortico-muscular coupling, the sonication duration was changed with different NTB (100, 150, 200, 250, 300). High-intensity and long duration ultrasound stimulation can produce 


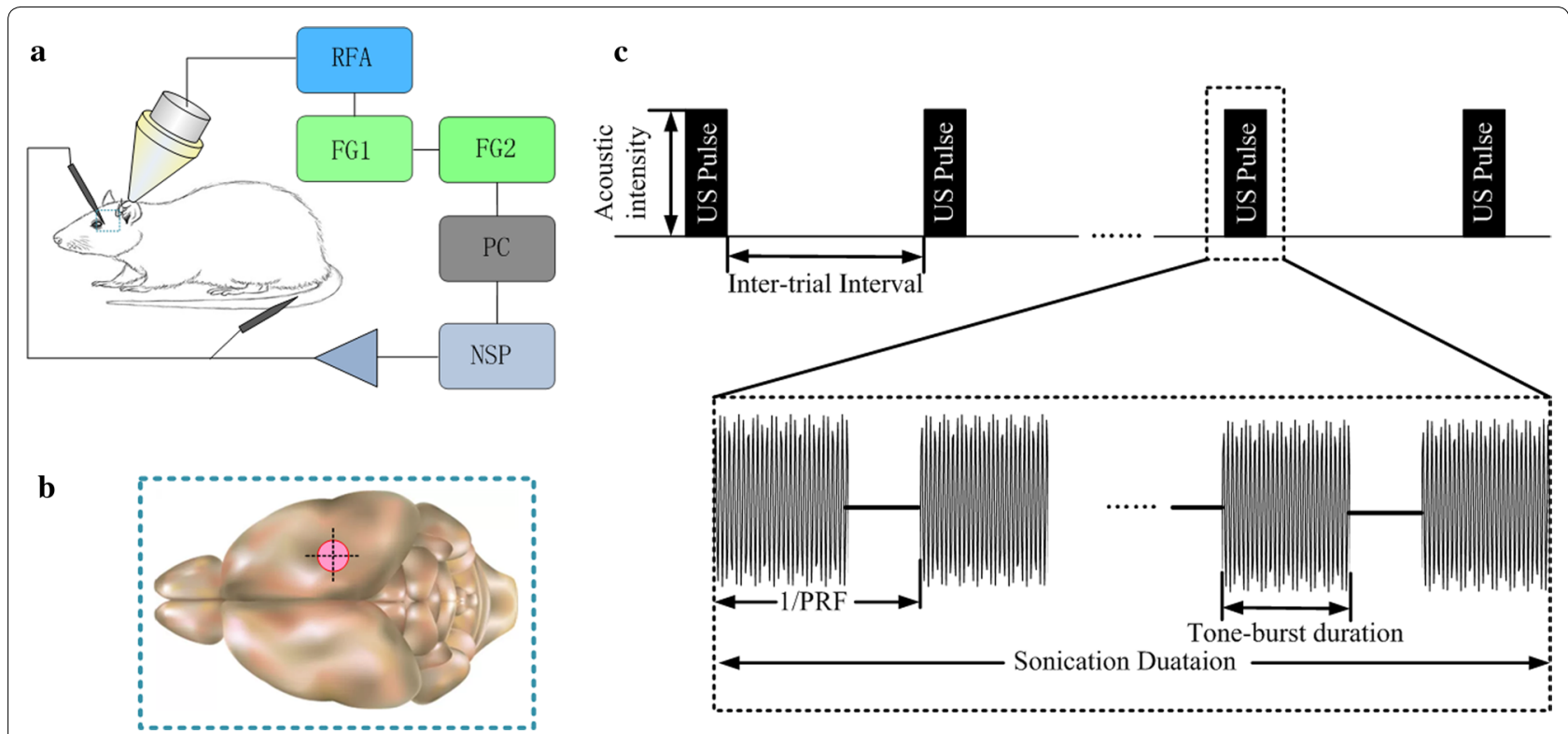

Fig. 1 The experimental system (a), sonication position (b) and parameters used for generating pTUS signal (c)

thermal effects and damage brain tissue [40]. Therefore, it is safe to use low-intensity pTUS with $\mathrm{NTB}=100,150$, 200, 250, 300 in the present study [39]. The pTUS signals were digitized at a sample rate of $30 \mathrm{kHz}$.

\section{Animal surgery and anesthesia}

Nine BALB/c mice (male, body weights $\sim 20$ g, Beijing Vital River Laboratory Animal Technology Co., Ltd. China) were used in this study. After anesthetized with sodium pentobarbital $(1 \%, 5 \mathrm{mg} / 100 \mathrm{~g}$, IP), mice were constrained on the stereotaxic apparatus (68002, 68030, RWD Co., China). Then, the fur covering the scalp was shaved and the skin was cleaned with physiological saline solution. The scalp of the mice was incised along the midline of the skull, and the exposed tissues and periosteum were cleaned carefully to expose the skull. Finally, the sonication site as illustrated in Fig. 1b, was determined by an atlas and a cranial window of $\sim 0.5 \times 0.5 \mathrm{~cm}$ was drilled to expose the brain tissue in the tail motor cortex. At the end of the experiment, mice were sacrificed with an overdose anesthetic (sodium pentobarbital, $1 \%$, $15 \mathrm{mg} / 100 \mathrm{~g}$, IP). All the experiment steps were approved with the Animal Ethics and Administrative Council of Yanshan University, Hebei Province, China.

\section{Data acquisition}

After the surgery procedure, a tungsten microelectrode was inserted into the tail motor cortex to acquire the LFPs signal, a fine-wire was inserted into tail muscle to acquire the EMG signal. When the anesthesia effect in mice was over, the LFPs and EMG signals were synchronously recorded at $2 \mathrm{kHz}$ using the same device. The angle between the pTUS and microelectrode was $\sim 60^{\circ}$. The acoustic collimator connected with the planar ultrasound transducer was aimed at the mice tail motor cortex. The ultrasonic wave passed through the acoustic collimator to stimulate the brain tissue for noninvasive neuromodulation.

\section{Data processing and analysis Data preprocessing}

To reject the artifacts in raw LFPs and EMG recordings, a notch filter was used to remove the power signal of $50 \mathrm{~Hz}$ and an adaptive high-pass filter was used to remove baseline drift. The LFPs and EMG was band-passed to $0.5-200 \mathrm{~Hz}$ and $10-200 \mathrm{~Hz}$, respectively. Then, the EMG was rectified. Finally, the LFPs and EMG before and after stimulation were cut in trials according to the pulse of TUS. After preprocessing, the LFPs, EMG and pTUS were shown in Fig. 2, were used subsequent analysis.

\section{Cortico-muscular coupling analysis by mutual information}

In this paper, the amount coupled information of cortico-muscular under pTUS was quantitively described by mutual information [28]. The LFPs and EMG were denoted as $x_{t}$ and $y_{t}$, respectively. The entropy of LFPs could be computed as following:

$$
H(L F P)=-\int_{x} p(x) \log (p(x)) d x
$$


where $p(x)$ is the probability density function of LFPs. The entropy of EMG can be calculated as the same way.

The joint entropy of LFPs and EMG is:

$$
H(L F P, E M G)=-\int_{x} \int_{y} p(x, y) \log (p(x, y)) d x d y
$$

where $p(x, y)$ is the joint probability density function of LFPs and EMG.

The mutual information between LFPs and EMG is:

$$
\begin{aligned}
M I(L F P, E M G) & =H(L F P)+H(E M G)-H(L F P, E M G) \\
& =\int_{x} \int_{y} p(x, y) \log \frac{p(x, y)}{p(x) p(y)}
\end{aligned}
$$

\section{Cortico-muscular coupling analysis by transfer entropy}

The directional interaction of CMC under pTUS was represented by transfer entropy [29]. Two time series $x_{t}$ and $y_{t}$ were approximated by Markov process, the transfer entropy from LFPs to EMG under pTUS can be written as follows:

$$
\begin{aligned}
T E_{L F P \rightarrow E M G} & =H\left(y_{t+1} \mid y_{t}^{n}\right)-H\left(y_{t+1} \mid x_{t}^{n}, y_{t}^{n}\right) \\
& =\sum_{y_{t+1}, y_{t}^{n}, x_{t}^{m}} p\left(y_{t+1}, y_{t}^{n}, x_{t}^{m}\right) \log \left(\frac{p\left(y_{t+1} \mid y_{t}^{n}, x_{t}^{m}\right)}{p\left(y_{t+1} \mid y_{t}^{n}\right)}\right)
\end{aligned}
$$

where $x_{t}^{m}=\left(x_{t}, \ldots, x_{t-m+1}\right)$ and $y_{t}^{n}=\left(y, \ldots, y_{t-n+1}\right)$, $m$ and $n$ are the orders of Markov process. $H\left(y_{t+1} \mid y_{t}^{n}\right)$ is the conditional entropy of EMG depending on the past values.

The two processes LFPs and EMG are reconstructed to a higher and same dimensional space. Thus, the formula of transfer entropy for two time series can be written as follows [41]:

$$
T E_{L E P \rightarrow E M G}=\sum_{y_{t+u}, y_{t}^{d}, x_{t}^{d}} p\left(y_{t+1}, y_{t}^{d}, x_{t}^{d}\right) \log \left(\frac{p\left(y_{t+u} \mid y_{t}^{d}, x_{t}^{d}\right)}{p\left(y_{t+u} \mid y_{t}^{n}\right)}\right)
$$

where $\quad x_{t}^{d}=\left(x_{t}, x_{t-\tau}, x_{t-2 \tau}, \ldots, x_{t-(d-1) \tau}\right) \quad$ and $y_{t}^{d}=\left(y_{t}, y_{t-\tau}, y_{t-2 \tau}, \ldots, y_{t-(d-1) \tau}\right)$. The $d, \tau$ and $u$ are the embedding dimension, embedding delay and the

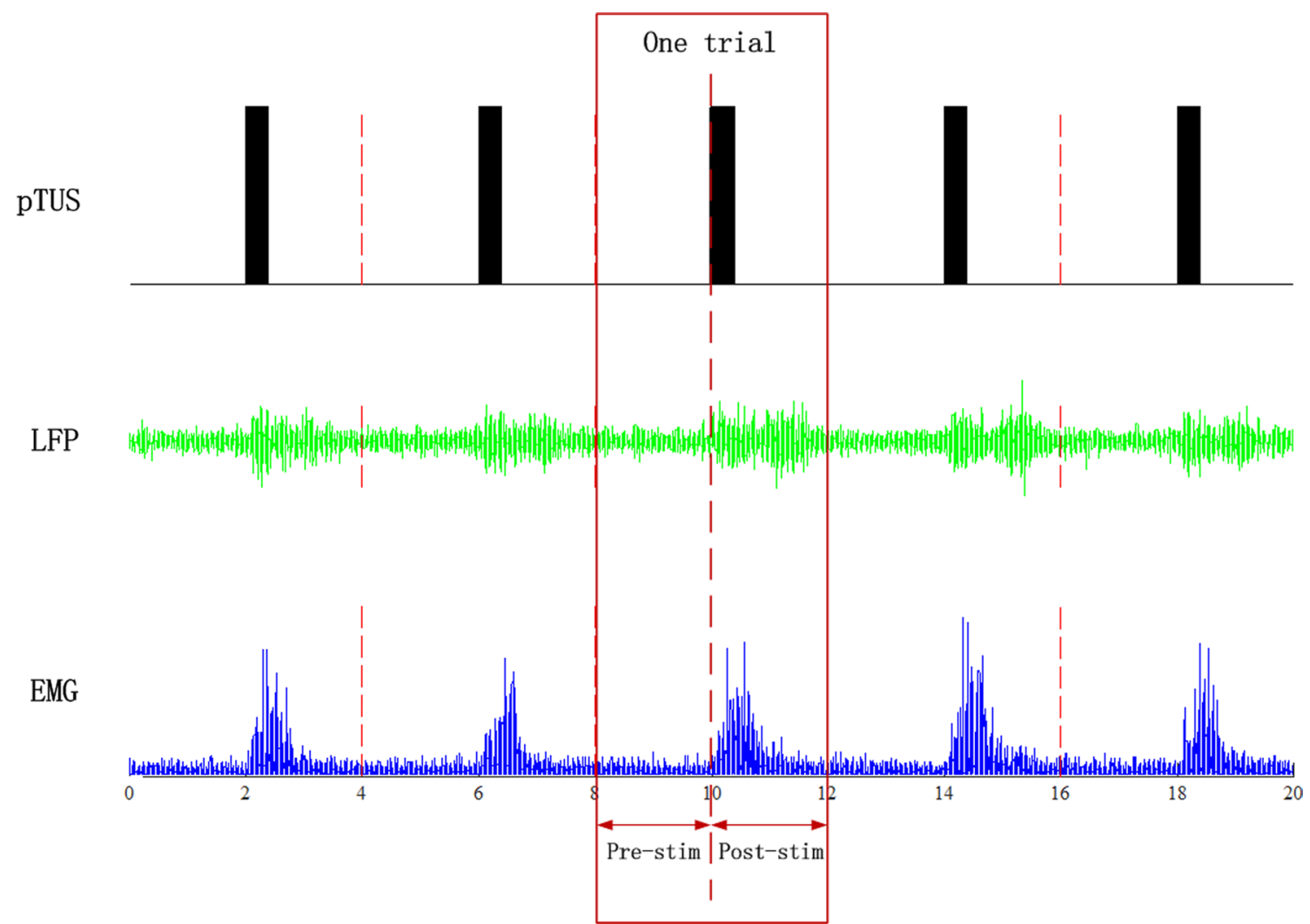

Fig. 2 pTUS and the LFPS and EMG signals after preprocessed. The 'Pre-stim' represents the time series recorded before stimulation. Reversely, 'Post-stim' represents the time series recorded after stimulation 
prediction time, respectively. The transfer entropy from EMG to LFPs is $T E_{E M G \rightarrow L F P}$ computed by the same process.

In this paper, the values of mutual information and transfer entropy were calculated using TRENTOOL toolbox [42]. Specifically, The embedding delay $(\tau)$ and embedding dimension $(d)$ for state space reconstruction were determined according to Ragwitz criterion [43]. The Kraskove-Stögbauere-Grassberger estimator and the nearest-neighbor search were applied to perform the TE estimation [44]. The number of neighbors $k$ was set to 4 as suggested in [45]. The prediction time $u$ was optimized in the range of $[10,49] \mathrm{ms}$ according to the influence of pTUS to EMG responses latency [13].

\section{Statistical analysis}

The differences between the TE/MI values of pre-stimulation and post-stimulation were statistically analyzed based on one-way repeated measures analysis of variance (rANOVA), and the differences between the TE values of the descending direction and ascending direction also performed by one-way rANOVA. The correlations between LFPs/EMG and MI/TE values at different NTB were determined using PEARSONs Correlation coefficient. The correlation was calculated using the MI/TE values and mean values of LFPs/EMG data in each trial. Significance level was set as $\mathrm{p}<0.05$. All the results of MI and TE were expressed as mean \pm S.D. SPSS 19.0 for windows (SPSS Inc., Chicago, IL, USA) was used for all statistical computations.

\section{Results}

\section{MI result}

To investigate the interaction information between motor cortex and tail muscle, the mean MI values between LFPs and EMG acquired from nine mice were calculated. Figure 3a shows the results of MI between LFPs and EMG before and after stimulation. Before the motor cortex was stimulated by the pTUS, the MI values between LFPs and EMG at different NTB were $0.0600 \pm 0.0040,0.0595 \pm 0$ $.0029,0.0610 \pm 0.0030,0.0627 \pm 0.0038,0.0630 \pm 0.0034$ (mean \pm S.D, $n=9$ ). After the motor cortex was stimulated by the pTUS, the MI values were $0.0649 \pm 0.0034,0.0651$ $\pm 0.0030,0.0716 \pm 0.0032,0.0732 \pm 0.0029,0.0719 \pm 0.0020$ (mean \pm S.D, $\mathrm{n}=9$ ). There were highly significant differences $(p<0.01$, one-way ANOVA) of MI between before and after stimulation in descending direction at $\mathrm{NTB}=200$, 250,300 cyc, while lower significant differences $(\mathrm{p}<0.05)$ of MI between before and after stimulation in ascending direction at $\mathrm{NTB}=150$ cyc, and no significant difference ( $p>0.05)$ between them when NTB $=100$ cyc.

To further explore the influence of pTUS at different NTB on MI values, we performed a significant test with the post-stimulation MI results. As shown in Fig. 3b, when $\mathrm{NTB}=200,250,300$, the MI results were significantly increased $(\mathrm{p}<0.05$, one-way ANOVA) compared with $\mathrm{NTB}=100$.

\section{TE result}

To study the changes of directional interaction information between motor cortex and tail muscle that was
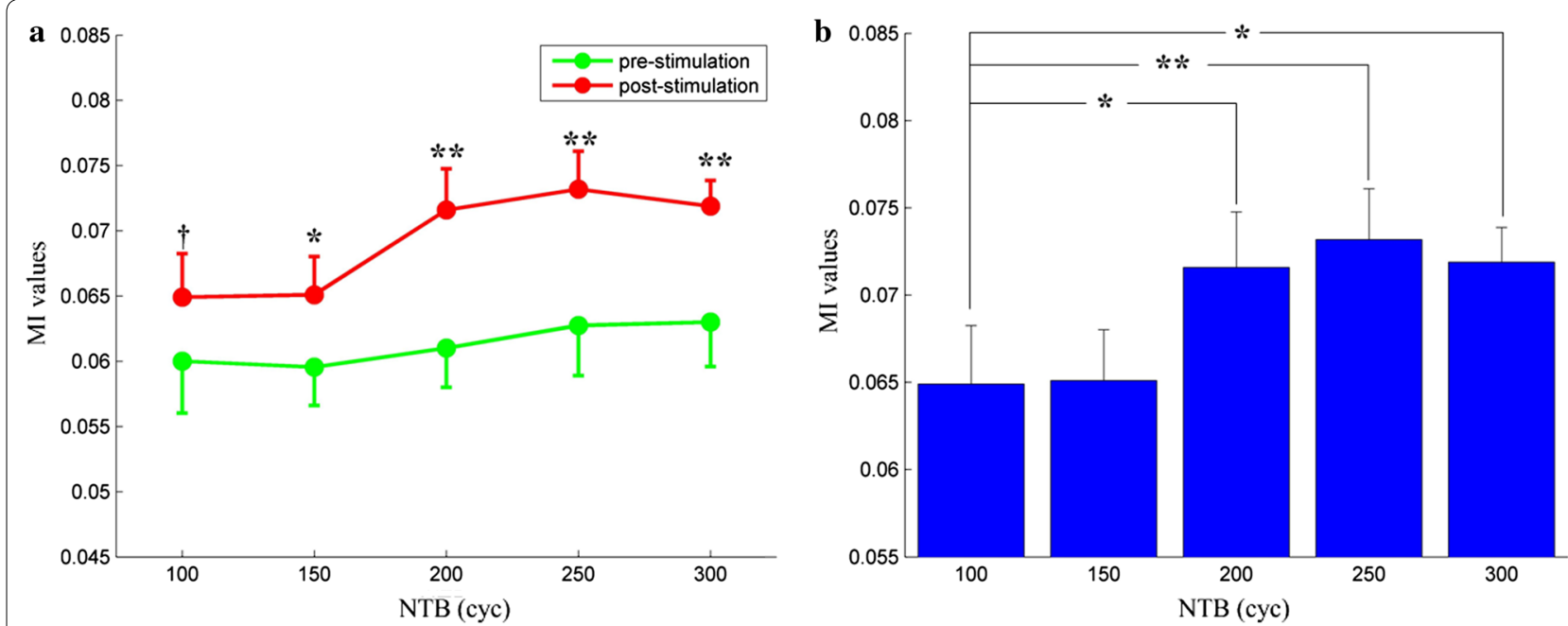

Fig. 3 The effects of pTUS to the mutual information between LFPs and EMG. a The results of MI before (green line) and after (red line) ultrasound stimulation. $\mathbf{b}$ The results of Ml under pTUS of NTB $=100,150,200,250,300 .{ }^{*} p<0.05,{ }^{* *} p<0.01,+p=0.05$ 
induced by pTUS with different NTB, we calculated the transfer entropy in both descending (from LFPs to EMG) and ascending (from EMG to LFPs) directions. Figure 4 shows the TE results in descending and ascending direction before and after stimulation. Before the motor cortex was stimulated by the pTUS, the TE values from LFPs to EMG at different NTB were $0.0327 \pm 0.0016,0.0329 \pm 0.0015,0.0329 \pm 0.0019,0.0335$ $\pm 0.0016,0.0333 \pm 0.0015($ mean \pm S.D, $\mathrm{n}=9)$. And the TE values from EMG to LFPs at different NTB were 0.0 $341 \pm 0.0012,0.0325 \pm 0.0011,0.0342 \pm 0.0013,0.0340 \pm$ $0.0014,0.0346 \pm 0.0018($ mean \pm S.D, $\mathrm{n}=9$ ).

After the motor cortex was stimulated by the pTUS, the $\mathrm{TE}$ values in descending direction were $0.0393 \pm 0.0021$ $, 0.0410 \pm 0.0018,0.0404 \pm 0.0019,0.0426 \pm 0.0021,0.0441$ \pm 0.0026 (mean \pm S.D, $n=9$ ). The TE values in ascending direction were $0.0382 \pm 0.0021,0.0377 \pm 0.0018,0.039$ $0 \pm 0.0019,0.0388 \pm 0.0016,0.0402 \pm 0.0015 \quad$ (mean \pm S.D, $\mathrm{n}=9$ ). Moreover, the TE values in both two directions were increased after the motor cortex was exposed to pTUS.

The significant analysis (the four lines at the top of Fig. 4) showed highly significant differences $(\mathrm{p}<0.01$, one-way rANOVA) of TE between before and after stimulation in descending direction (Line 4), while lower significant differences $(p<0.05$, one-way rANOVA) of TE between before and after stimulation in ascending direction (Line 3). Additionally, there were three significant differences ( $\mathrm{NTB}=150,250,300 \mathrm{cyc}$ ) between the TE in descending and ascending direction after stimulation (Line 2), while no significant difference between them before stimulation (Line 1).

The effect of different parameters of pTUS to the transfer entropy between LFPs and EMG was shown in Fig. 5. In Fig. $5 \mathrm{a}$, when $\mathrm{NTB}=200,250,300 \mathrm{cyc}$, the TE values in descending direction were significantly increased $(\mathrm{p}<0.05$, one-way rANOVA) compared with NTB $=100$ cyc, where the most significant increase $(\mathrm{p}<0.01)$ was

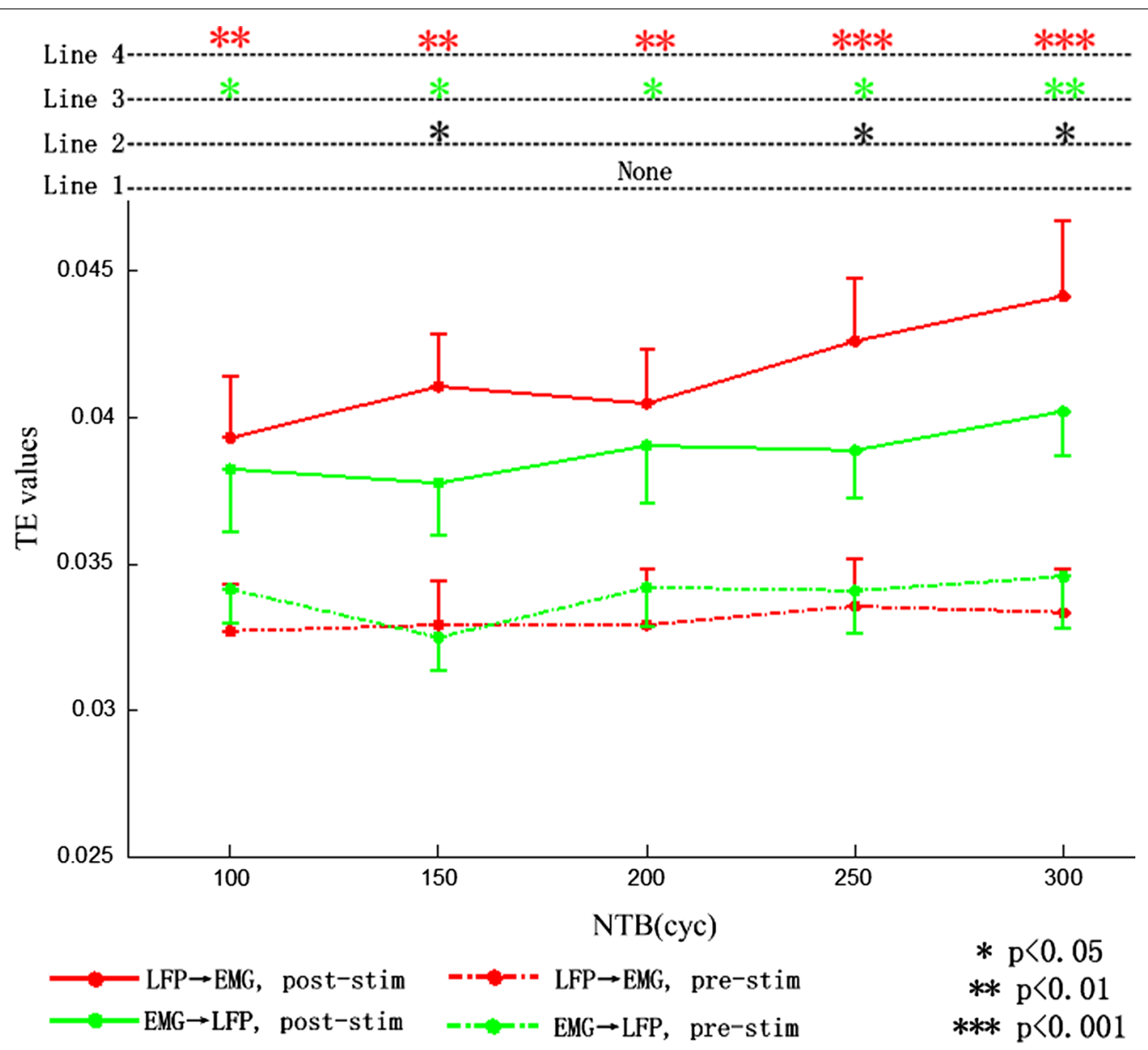

Fig. 4 The TE values between LFPs and EMG before and after stimulation. The "Line 1" showed the significant level between descending and ascending direction before ultrasound stimulation, the "Line 2" was after ultrasound stimulation. The Line 3 and Line 4 indicated the significant level between before and after stimulation of ascending direction (from EMG to LFPs) and descending direction (from LFPs to EMG), respectively 
in $\mathrm{NTB}=300$ cyc. In Fig. 5b, when NTB $=250,300$ cyc, the TE values in ascending direction were significantly increased $(\mathrm{p}<0.05)$ compared with $\mathrm{NTB}=100$ cyc.

\section{Discussion}

Ultrasound stimulation has emerged as a potential approach that can address the defects faced by modern neuromodulation technologies [7, 46], which can be applied noninvasively to activate or modulate the activity of targeted brain regions [16, 47, 48]. Recent years, many studies found evidently motor responses in animals by activating the primary motor cortex using the transcranial ultrasound $[5,13,14,49,50]$, where the induced movement was all measured by EMG signals. However, both motor command from brain and feedback from muscle are involved in effective movement control [22, 23], and there is a coupled relationship between the cortical oscillation and muscle activation [51, 52]. To our knowledge, there are still a lack of evidence that assessed the neuromodulatory effect of pTUS from the neural circuit of motor-control system view. Thus, in this study, we considered applying the cortico-muscular coupling to evaluate the effect of pTUS with different number of tone bursts (NTB). Since CMC has been applied to assess the movement response induced by other neuromodulation techniques, such as transcranial magnetic stimulation (TMS), transcranial alternating current stimulation (tACS) and deep-brain stimulation (DBS) [53-55], we assume that the CMC could serve as a promising tool for the assessment of ultrasound neuromodulation.

Intention of the present study is to elucidate the effect of pTUS with different number of tone bursts (NTB) using CMC. We recorded LFPs and EMG evoked by pTUS in mice's motor cortex and tail muscle. As shown in Fig. 2, the amplitude of LFPs and EMG signals increased after stimulation. It means that the neural activity of motor cortex and the contralateral muscle could be altered by pTUS, which supports the previous studies of pTUS $[5,13,49]$. Then, we analyzed the coupling relationships between these two kind of signals using mutual information and transfer entropy.

We can see that both the TE and MI values between LFPs and EMG signals could be significantly increased with pTUS. These results indicated that the CMC between motor cortex and tail muscle could be enhanced by pTUS. Although the reason of the enhancement of CMC induced by pTUS is still unclear, the pTUS-induced EMG response [13] and the cortical excitement [56] might be related to this phenomenon, as significant correlations $(\mathrm{p}<0.05)$ between the mean amplitude of the recorded signals (LFPs and EMG) and MI values could be observed when NTB $=100,200,250,300$ (Table 1), where the quality of the correlation was expressed by $\rho$, and the significant level was expressed by $\mathrm{p}$.

The MI results revealed that the CMC in the sensory and motor system could be enhanced by pTUS (Fig. 3a). The TE results suggested that the CMC in descending direction could be significantly higher than that in ascending direction after stimulation (Fig. 4). It suggested that the neural pathways responded for motor command would transmit more information than the feedback pathway due to the effect of pTUS. Correlation analysis (Table 1$)$ showed a highly significant correlation $(\mathrm{p}<0.01)$ between the mean LFPs amplitude and the TE values in
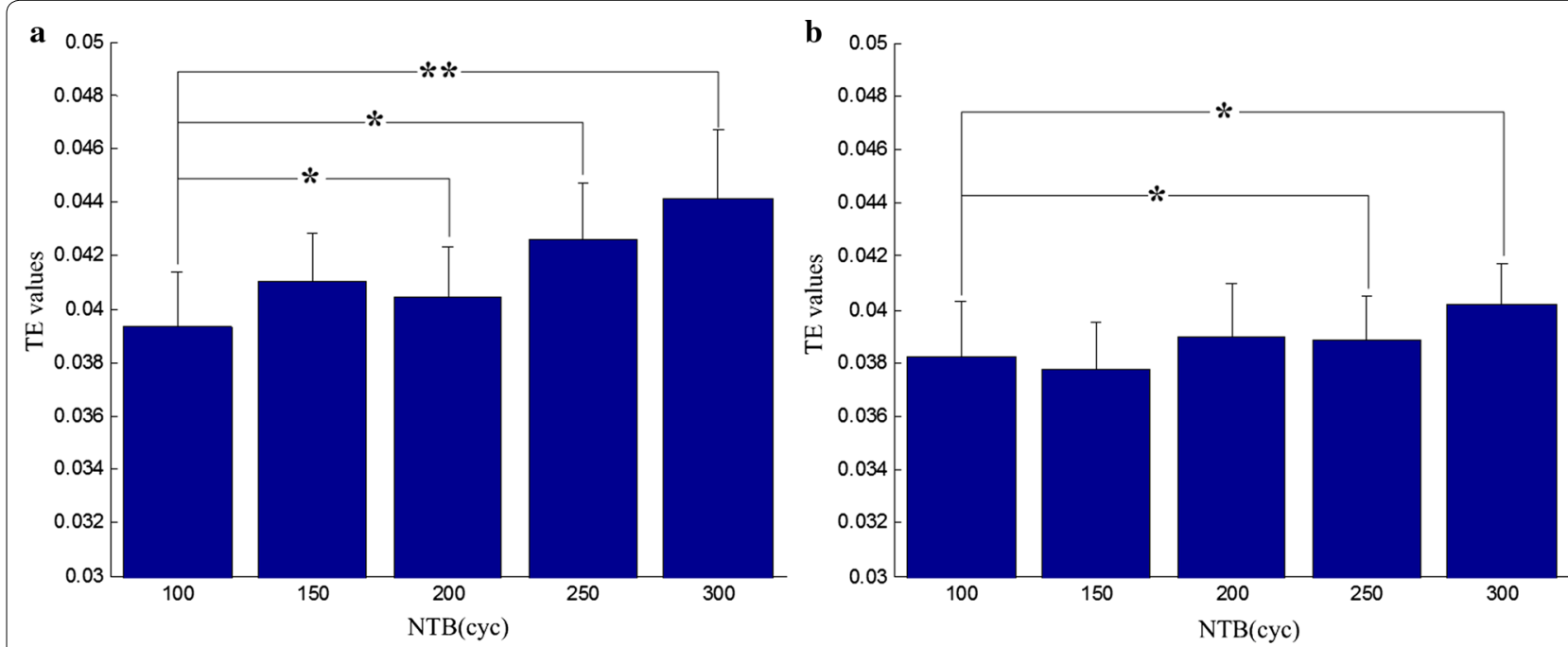

Fig. 5 The effects of pTUS with different NTB on the transfer entropy between LFPs and EMG. a The results of transfer entropy of descending direction (from LFPs to EMG). b The results of transfer entropy of ascending direction (from EMG to LFPs). "** denotes $p<0.05$, "***" denotes $p<0.01$ 
Table 1 Results of correlation analysis

\begin{tabular}{lllll}
\hline NTB & Correlation LFPs and MI & Correlation EMG and MI & $\begin{array}{l}\text { Correlation LFPs and TE } \\
\text { (LFPs } \rightarrow \text { EMG) }\end{array}$ & $\begin{array}{l}\text { Correlation LFPs and TE } \\
\text { (EMG } \rightarrow \text { LFPs) }\end{array}$ \\
\hline 100 & $\rho^{2}=0.032$ & $\rho^{2}=0.014$ & $\rho^{2}=0.010$ & $\rho^{2}=0.012$ \\
& $p=0.035$ & $p=0.043$ & $p=0.0061$ & $p=0.058$ \\
150 & $\rho^{2}=0.010$ & $\rho^{2}=0.001$ & $\rho^{2}=0.023$ & $\rho^{2}=0.002$ \\
& $p=0.062$ & $p=0.086$ & $p=0.0046$ & $p=0.084$ \\
200 & $\rho^{2}=0.036$ & $\rho^{2}=0.032$ & $\rho^{2}=0.026$ & $\rho^{2}=0.0001$ \\
& $p=0.026$ & $p=0.036$ & $p=0.0043$ & $p=0.096$ \\
250 & $\rho^{2}=0.036$ & $\rho^{2}=0.036$ & $\rho^{2}=0.090$ & $\rho^{2}=0.003$ \\
& $p=0.024$ & $p=0.034$ & $p=0.0015$ & $p=0.081$ \\
& $\rho^{2}=0.040$ & $\rho^{2}=0.023$ & $\rho^{2}=0.292$ & $\rho^{2}=0.026$ \\
\end{tabular}

descending direction (LFPs $\rightarrow$ EMG) $(\mathrm{NTB}=150,200$, $250,300)$, and a poor correlation $(\mathrm{p}>0.05)$ in ascending direction (LFPs $\rightarrow$ EMG). The results revealed that the transferred information from the brain to muscle might be facilitated by the excitement of neural activity in motor cortex. It suggested that CMC could serve as a more useful tool for evaluating the effect of pTUS in motor cortex, which could not only assess the pTUSinduced motor responses as previous studies did by using LFP and EMG [12-14], but also reveal the information interaction between motor cortex and muscle in motor system. The mechanism of cortical excitement evoked by pTUS is still debated $[57,58]$. In general, cavitation of neural membrane is known as the critical factor for eliciting neuromodulatory efficacy, which has been confirmed in cellular-scale and in vivo [50]. Recent findings revealed an indirect auditory mechanism for ultrasound-induced cortical activity and movement $[57,58]$. And we speculate that the no-task experimental condition in mouse, which was different from human [35], might also result in the lower CMC in ascending direction compared with another direction. Overall, the results in this study revealed that MI could be applied to quantitatively estimate the integral CMC between motor cortex and contralateral muscle during pTUS in mouse. And TE could be used to analyze the change of directional interaction information between them. Moreover, the CMC estimated by MI and TE could increase with the increasing of NTB (NTB $=100,150,200,250,300)$ (Figs. 3b, 5). As the sonication duration depends a lot on the NTB, this study reveals a positive correlation between CMC and stimulus duration. It also supports that the longer stimulus duration increases the probability of motor response [26, 39].

Furthermore, previous studies showed that the motor response induce by other brain stimulation techniques such as TMS, tACS, DBS could be assessed by CMC in human [53-55], especially in patients with motor dysfunction diseases. For example, the CMC of Parkinson's disease (PD) could be modulated by DBS [59]. And pTUS had shown cerebral protection effect for stroke [10]. In this study, the CMC in descending direction is significantly increased and higher than the ascending direction after ultrasound stimulation (Fig. 5a). As it is generally believed that the impairment in neural-pathway of the descending direction is the main cause of stroke [35], applying CMC into the evaluation of pTUS neuromodulation may provide an evidence for understanding the mechanism of pTUS in stroke rehabilitation. Moreover, the MI and TE methods, which quantified CMC, could be used for measuring the effect of ultrasound stimulation and optimizing the ultrasonic duration.

Since this study only explored the influence of pTUS on the CMC in healthy mouse, we plan to extend this work to stroke or PD mice to investigate whether the abnormal CMC in those diseases can be improved by pTUS. Additionally, a previous study suggested that the ultrasound-induced EMG signals in mouse could increase as a function of both the ultrasound intensity and sonication duration [26]. Our study only investigated the effect of sonication duration to $\mathrm{CMC}$, perhaps other parameters such as ultrasound intensity, frequency or number of cycles could also produce modulation effects to CMC. The influence of the ultrasound parameters to $\mathrm{CMC}$ could be systematically studied in the next step.

\section{Conclusion}

In this study, the neuromodulatory effect of pulsed transcranial ultrasound was analyzed by the cortico-muscular coupling between motor cortex and tail muscle in mice, which was quantified using the transfer entropy and mutual information algorithms. The results of MI 
and TE showed that the CMC between motor cortex and tail muscle was significantly increased by pTUS, and the CMC in descending direction could be significantly higher than that of ascending direction after ultrasound stimulation. Furthermore, by increasing the NTB, the CMC between motor cortex and tail muscle could also be significantly enhanced. Since the CMC is a promising tool for movement evaluation, it suggests that pTUS might influence the motor function of mice. This study demonstrates for the first time, using MI and TE method, the CMC can be used for quantitatively evaluating the effect of different sonication durations of pTUS-induced movement, which might provide a new insight into the effect of pTUS neuromodulation in motor cortex.

\begin{abstract}
Abbreviations
pTUS: pulsed transcranial ultrasound stimulation; EMG: electromyograms; CMC: cortico-muscular coupling; NTB: number of tone bursts; MI: mutual information; TE: transfer entropy; LFPs: local field potentials; MEG: magnetoencephalogram; EEG: electroencephalogram; FG: function generators; RFA: radio frequency power amplifier; NSP: neural signal processor; Al: acoustic intensity; NC: number of acoustic cycles per pulse; PRF: pulse repetition frequency; ITI: inter trial interval; TMS: transcranial magnetic stimulation; tACS: transcranial alternating current stimulation; DBS: deep-brain stimulation; PD: Parkinson's disease.
\end{abstract}

\section{Authors' contributions}

PX, SZ and YY were responsible for experimental design, analysis and manuscript preparation. XW and YW were responsible for experimental data collection, statistical analysis and manuscript revision. All authors read and approved the final manuscript.

\section{Acknowledgements}

None.

\section{Competing interests}

The authors declare that they have no competing interests.

\section{Availability of data and materials}

The dataset analyzed during the current study are available. The amount of data involved in the paper is too large to be individually uploaded. If the data is needed, please contact the corresponding author on reasonable request.

\section{Consent to publish}

Not applicable.

\section{Ethics approval and consent to participate}

All the experiments with mice were approved by the Animal Ethics and Administrative Council of Yanshan University, Hebei Province, China (Permit No.: 16204).

\section{Funding}

This work was supported by National Nature Science Foundation of China under Grants 61673336, and Natural Science Foundation of Hebei, China under Grants F2015203372. The funders do not participate in the experimental research, or preparation the manuscript.

\section{Publisher's Note}

Springer Nature remains neutral with regard to jurisdictional claims in published maps and institutional affiliations.

Received: 18 February 2018 Accepted: 21 September 2018 Published online: 03 October 2018

\section{References}

1. Lewis PM, Thomson RH, Rosenfeld JV, Fitzgerald PB. Brain neuromodulation techniques: a review. Neurosci Rev J Bringing Neurobiol Neurol Psychiatry. 2016;22(4):406-21

2. Leinenga G, Götz J. Scanning ultrasound removes amyloid- $\beta$ and restores memory in an Alzheimer's disease mouse model. Sci Transl Med. 2015:7(278):278ra233.

3. Mueller JK, Ai L, Bansal P, Legon W. Numerical evaluation of the skull for human neuromodulation with transcranial focused ultrasound. J Neural Eng. 2017;14(6):066012.

4. Panczykowski DM, Rd ME, Friedlander RM. Transcranial focused ultrasound modulates the activity of primary somatosensory cortex in humans. Neurosurgery. 2014;74(6):322.

5. Yoo SS, Bystritsky A, Lee JH, Zhang Y, Fischer K, Min BK, Mcdannold NJ, Pascualleone A, Jolesz FA. Focused ultrasound modulates region-specific brain activity. Neuroimage. 2011;56(3):1267-75.

6. Bystritsky A, Korb AS, Douglas PK, Cohen MS, Melega WP, Mulgaonkar AP, Desalles A, Min BK, Yoo SS. A review of low-intensity focused ultrasound pulsation. Brain Stimul. 2011;4(3):125.

7. Naor O, Krupa S, Shoham S. Ultrasonic neuromodulation. J Neural Eng. 2016;13(3):031003.

8. Tyler WJ, Yusuf T, Michael F, Tauchmann ML, Olson EJ, Cassondra M. Remote excitation of neuronal circuits using low-intensity, low-frequency ultrasound. PLoS ONE. 2008;3(10):e3511.

9. Legon W, Bansal P, Tyshynsky R, Ai L, Mueller JK. Transcranial focused ultrasound neuromodulation of the human primary motor cortex. Sci Rep. 2018;8:10007.

10. Li H, Sun J, Zhang D, Omiremayor D, Lewin PA, Tong S. Low-intensity $\left(400 \mathrm{~mW} / \mathrm{cm}^{2}, 500 \mathrm{kHz}\right.$ ) pulsed transcranial ultrasound preconditioning may mitigate focal cerebral ischemia in rats. Brain Stimul. 2017:10(3):695-702.

11. Yuan Y, Yan J, Ma Z, Li X. Noninvasive focused ultrasound stimulation can modulate phase-amplitude coupling between neuronal oscillations in the rat hippocampus. Front Neurosci. 2016;10(191):348.

12. Mueller J, Legon W, Opitz A, Sato TF, Tyler WJ. Transcranial focused ultrasound modulates intrinsic and evoked EEG dynamics. Brain Stimul. 2014;7(6):900-8.

13. Tufail Y, Matyushov A, Baldwin N, Tauchmann ML, Georges J, Yoshihiro A, Tillery SI, Tyler WJ. Transcranial pulsed ultrasound stimulates intact brain circuits. Neuron. 2010;66(5):681.

14. King RL, Brown JR, Pauly KB. Localization of ultrasound-induced in vivo neurostimulation in the mouse model. Ultrasound Med Biol. 2014:40(7):1512-22.

15. Tufail Y, Yoshihiro A, Pati S, Li MM, Tyler WJ. Ultrasonic neuromodulation by brain stimulation with transcranial ultrasound. Nat Protoc. 2011;6(9):1453.

16. Legon W, Sato TF, Opitz A, Mueller J, Barbour A, Williams A, Tyler WJ. Transcranial focused ultrasound modulates the activity of primary somatosensory cortex in humans. Nat Neurosci. 2014;17(2):322.

17. Guo T, Li H, Lv Y, Lu H, Niu J, Sun J, Yang GY, Ren C, Tong S. Pulsed transcranial ultrasound stimulation immediately after the ischemic brain injury is neuroprotective. IEEE Trans Biomed Eng. 2015;62(10):2352-7.

18. Conway BA, Halliday DM, Farmer SF, Shahani U, Maas P, Weir Al, Rosenberg JR. Synchronization between motor cortex and spinal motoneuronal pool during the performance of a maintained motor task in man. J Physiol. 1995;489(3):917

19. Baker SN, Olivier E, Lemon RN. Coherent oscillations in monkey motor cortex and hand muscle EMG show task-dependent modulation. J Physiol. 1997;501 (Pt 1)(1):225.

20. Kakei S, Hoffman DS, Strick PL. Muscle and movement representations in the primary motor cortex. Science. 1999;285(5436):2136-9.

21. Mehrkanoon S, Breakspear M, Boonstra TW. The reorganization of corticomuscular coherence during a transition between sensorimotor states. Neuroimage. 2014;100:692-702.

22. Mima T, Matsuoka T, Hallett M. Information flow from the sensorimotor cortex to muscle in humans. Clin Neurophys Off J Int Fed Clin Neurophys. 2001;112(1):122-6.

23. Kilner JM, Baker SN, Salenius S, Hari R, Lemon RN. Human cortical muscle coherence is directly related to specific motor parameters. J Neurosci Off J Soc Neurosci. 2000;20(23):8838. 
24. Von Carlowitz-Ghori K, Bayraktaroglu Z, Hohlefeld FU, Losch F, Curio G, Nikulin W. Corticomuscular coherence in acute and chronic stroke. Clin Neurophys Off J Int Fed Clin Neurophysiol. 2014;125(6):1182-91.

25. Mehic E. Increased anatomical specificity for neuromodulation using modulated focused ultrasound. PLoS ONE. 2014;9(2):e86939.

26. King RL, Brown JR, Newsome WT, Pauly KB. Effective parameters for ultrasound-induced in vivo neurostimulation. Ultrasound Med Biol. 2013;39(2):312-31.

27. Jin $\mathrm{SH}$, Lin $\mathrm{P}$, Hallett M. Linear and nonlinear information flow based on time-delayed mutual information method and its application to corticomuscular interaction. Clin Neurophys Off J Int Fed Clin Neurophys. 2010;121(3):392-401.

28. Viola P, lii WMW. Alignment by maximization of mutual information. Int J Comput Vis. 1997;24(2):137-54.

29. Schreiber T. Measuring information transfer. Phys Rev Lett. 2000;85(2):461.

30. Vicente R, Wibral M, Lindner M, Pipa G. Transfer entropy-a model-free measure of effective connectivity for the neurosciences. J Comput Neurosci. 2011;30(1):45-67.

31. Zhou S, Xie P, Chen X, Wang Y, Zhang Y, Du Y. Optimization of relative parameters in transfer entropy estimation and application to corticomuscular coupling in humans. J Neurosci Methods. 2018. https://doi. org/10.1016/j.jneumeth.2018.07.004.

32. So WK, Yang L, Jelfs B, She Q, Wong SW, Mak JN, Chan RH: Cross-frequency information transfer from EEG to EMG in grasping. In: Engineering in medicine and biology society. 2016. p. 4531.

33. Chen CC, Hsieh JC, Wu YZ, Lee PL, Chen SS, Niddam DM, Yeh TC, Wu YT. Mutual-information-based approach for neural connectivity during selfpaced finger lifting task. Hum Brain Mapp. 2008;29(3):265

34. Mima T, Steger J, Schulman AE, Gerloff C, Hallett M. Electroencephalographic measurement of motor cortex control of muscle activity in humans. Clin Neurophys Off J Int Fed Clin Neurophys. 2000;111(2):326.

35. Witham CL, Riddle CN, Baker MR, Baker SN. Contributions of descending and ascending pathways to corticomuscular coherence in humans. J Physiol. 2011;589(15):3789-800.

36. Ouyang G, Li X. Estimating coupling direction between neuronal populations. Neuroimage. 2010;52(2):497-507.

37. Vakorin VA, Bratislav M, Olga K, Randal MIA. Empirical and theoretical aspects of generation and transfer of information in a neuromagnetic source network. Front Syst Neurosci. 2011;5:96

38. Mueller JK, Ai L, Bansal P, Legon W. Computational exploration of wave propagation and heating from transcranial focused ultrasound for neuromodulation. J Neural Eng. 2016;13(5):056002.

39. Kim H, Chiu A, Lee SD, Fischer K, Yoo SS. Focused ultrasound-mediated non-invasive brain stimulation: examination of sonication parameters. Brain Stimul. 2014;7(5):748-56.

40. Dalecki D. Mechanical bioeffects of ultrasound. Ann Rev Biomed Eng. 2004;6(1):229.

41. Wibral M, Rahm B, Rieder M, Lindner M, Vicente R, Kaiser J. Transfer entropy in magnetoencephalographic data: quantifying information flow in cortical and cerebellar networks. Prog Biophys Mol Biol. 2011;105(1-2):80-97.

42. Lindner M, Vicente R, Priesemann V, Wibral M. TRENTOOL: a Matlab open source toolbox to analyse information flow in time series data with transfer entropy. BMC Neurosci. 2011;12(1):119.
43. Ragwitz M, Kantz H. Markov models from data by simple nonlinear time series predictors in delay embedding spaces. Phys Rev E Stat Nonlinear Soft Matter Phys. 2002;65(5 Pt 2):056201.

44. Wibral M, Pampu N, Priesemann V, Siebenhühner F, Seiwert H, Lindner M, Lizier JT, Vicente R. Measuring information-transfer delays. PLOS One. 2013;8(2):e55809.

45. Kraskov A, Gbauer StH, Grassberger P. Estimating mutual information Phys Rev E Stat Nonlinear Soft Matter Phys. 2003;69(6 Pt 2):066138.

46. Ye J, Tang S, Meng L, Li X, Wen X, Chen S, Niu L, Li X, Qiu W, Hu H, et al. Ultrasonic control of neural activity through activation of the mechanosensitive channel MscL. Nano Lett. 2018;18(7):4148-55.

47. Lee W, Kim H, Jung Y, Song IU, Yong AC, Yoo SS. Image-guided transcranial focused ultrasound stimulates human primary somatosensory cortex. Sci Rep. 2015;5:8743.

48. Lee W, Kim HC, Jung Y, Yong AC, Song IU, Lee JH, Yoo SS. Transcranial focused ultrasound stimulation of human primary visual cortex. Sci Rep. 2016;6:34026

49. Legon W, Ai L, Bansal P, Mueller JK. Neuromodulation with single-element transcranial focused ultrasound in human thalamus. Hum Brain Mapp. 2018:39(5):1995-2006.

50. Ye PP, Brown JR, Pauly KB. Frequency dependence of ultrasound neurostimulation in the mouse brain. Ultrasound Med Biol. 2016:42(7):1512-30.

51. Murase N, Duque J, Mazzocchio R, Cohen LG. Influence of interhemispheric interactions on motor function in chronic stroke. Ann Neurol. 2004;55(3):400.

52. Schulz H, Ubelacker T, Keil J, Müller N, Weisz N. Now I am ready-now i am not: the influence of pre-TMS oscillations and corticomuscular coherence on motor-evoked potentials. Cereb Cortex. 2014;24(7):1708-19.

53. Sağlam M, Matsunaga $K$, Murayama N, Hayashida $Y$, Huang $Y Z$, Nakanishi R. Parallel inhibition of cortico-muscular synchronization and corticospinal excitability by theta burst TMS in humans. Clin Neurophys Off J Int Fed Clin Neurophys. 2008;119(12):2829-38.

54. Wach C, Krause V, Moliadze V, Paulus W, Schnitzler A, Pollok B. The effect of $10 \mathrm{~Hz}$ transcranial alternating current stimulation (tACS) on corticomuscular coherence. Front Hum Neurosci. 2013;7(5):511.

55. Beuter A, Lefaucheur JP, Modolo J. Closed-loop cortical neuromodulation in Parkinson's disease: an alternative to deep brain stimulation? Clin Neurophys Off J Int Fed Clin Neurophys. 2014;125(5):874-85.

56. Kamimura HAS, Wang $\mathrm{S}$, Chen $\mathrm{H}$, Wang Q, Aurup C, Acosta C, Carneiro $\mathrm{AAO}$, Konofagou EE. Focused ultrasound neuromodulation of cortical and subcortical brain structures using 1.9 MHz. Med Phys. 2016;43(10):5730.

57. Guo H, Mark Hamilton II, Offutt SJ, Gloeckner CD, Li T, Kim Y, Legon W, Alford JK, Lim HH. Ultrasound produces extensive brain activation via a cochlear pathway. Neuron. 2018;98(5):1020-30.e4

58. Sato T, Shapiro MG, Tsao DY. Ultrasonic neuromodulation causes widespread cortical activation via an indirect auditory mechanism. Neuron. 2018;98(5):1031.

59. Airaksinen K, Mäkelä JP, Nurminen J, Luoma J, Taulu S, Ahonen A, Pekkonen E. Cortico-muscular coherence in advanced Parkinson's disease with deep brain stimulation. Clin Neurophys Off J Int Fed Clin Neurophys. 2015;126(4):748.

Ready to submit your research? Choose BMC and benefit from

- fast, convenient online submission

- thorough peer review by experienced researchers in your field

- rapid publication on acceptance

- support for research data, including large and complex data types

- gold Open Access which fosters wider collaboration and increased citations

- maximum visibility for your research: over 100M website views per year

At BMC, research is always in progress.

Learn more biomedcentral.com/submissions 